\title{
Electromagnetic radiation related to dislocation dynamics in a seismic preparation zone
}

\author{
Roman Teisseyre and Tomasz Ernst \\ Institute of Geophysics, Polish Academy of Sciences, Warszawa, Poland
}

\begin{abstract}
Electromagnetic emission is observed frequently before earthquakes as high noise level in VLF and ULF bands. We present theoretical considerations on electromagnetic radiation caused by dislocation dynamics in the preseismic micro-sources (micro-crackings) located in an earthquake preparation zone. Some of these micro-sources could be located near the ground surface and their electromagnetic signals could be accessible in some recording stations. The examples of the numerically simulated induction and radiation fields are given and one example of the observed radio-noise recording is shown.
\end{abstract}

Key words stress evolution - instabilities - charge dislocations - electromagnetic emission

\section{Introduction}

The preseismic micro-source activity may be followed by a rebound motion resulting in the main break: the earthquake. The dynamics of micro-sources is related to the accelerated motion of dislocations and to coalescences of the opposite dislocation arrays (micro-cracks). The theory of stress and dislocation evolution is based on the equation of motion with the self-stress field (continuum with defect distribution) on the slipfault plane; this equation is supplemented with the source/sink function entering in a similar way as the body forces in the equation of motion for displacements. This theory of stress evolution in a pre-seismic zone combined with the physics

Mailing address: Prof. Roman Teisseyre, Polish Academy of Sciences, Institute of Geophysics, ul. Ks. Janusza 64, 01-402 Warszawa, Poland; e-mail: rt@igf.edu.pl of the charge dislocations leads to precursory electromagnetic emission.

\section{Evolution of stresses}

Most breaks start along the pre-existing faults or micro-faults. Considering a plane problem containing a fault, we may assume that the rigidity on a fault $\mu^{*}$ is smaller than the bulk value $\mu$ of the surrounding rocks: $\mu^{*}<\mu$. For the boundary between the fault zone and the intact rocks $y=0$, we can take the stressdislocations relation (see further on)

$$
\alpha=\frac{1}{\mu} \partial S / \partial x
$$

which leads us to the condition $\alpha^{*} \mu^{*}=\alpha \mu$. This is due to the continuity of tangent stress derivatives. We obtain a jump of dislocation density on a fault:

$$
\alpha^{*}>\alpha
$$

The preseismic micro-source activity may be 
followed by a rebound motion resulting in a main break: earthquake.

The dynamic processes on a fault, or on micro-faults, can be described by the equation of motion for stresses including the interaction with the self-stress fields (dislocation stresses). We follow the results obtained in the papers by Teisseyre (1996, 2001a,b) and Teisseyre and Yamashita (1999). According to Kossecka and DeWitt (1977), the motion equation is expressed by the balance of divergence of elastic stresses and elastic acceleration. Elastic acceleration is introduced as a difference between the total acceleration and self (plastic) acceleration (the total acceleration can be expressed by time derivatives of displacements). We obtain in this way the motion equation for the elastic continuum with defects

$$
\begin{gathered}
\operatorname{div} \boldsymbol{S}=\rho \frac{\mathrm{d}}{\mathrm{d} t} \boldsymbol{v}+\frac{\mathrm{d} \Pi}{\mathrm{d} t} \\
\operatorname{div} \boldsymbol{S}=\rho \frac{\mathrm{d}^{2}}{\mathrm{~d} t^{2}} \boldsymbol{u}-\rho \frac{\mathrm{d}}{\mathrm{d} t} \boldsymbol{v}^{s}+\frac{\partial \Pi}{\partial t}
\end{gathered}
$$

where $\boldsymbol{S}$ are the elastic stresses, $v$ is the elastic velocity, $\boldsymbol{u}$ is the total displacement, $v^{S}$ is the self/ plastic velocity (total velocity: $\dot{\boldsymbol{u}}=v+v^{S}$ ), $\Pi$ is the source/sink function ( $\dot{\Pi}$ corresponds to a body force).

In $1 \mathrm{D}$, a dislocation density $\alpha$ is related to stresses $S$ on a fault and also to a slip $\delta$ as follows:

$$
\alpha=\frac{1}{\mu} \nabla S, \quad \alpha=\frac{1}{\Lambda} \nabla \delta, \quad \delta=\frac{\Lambda}{\mu}\left(S-S^{F}\right)
$$

where $S^{F}$ is the resistance stress (friction stress in the fracture phase) related to slip progress on fault, $\Lambda$ is here a certain reference thickness: when computing dislocation density related to slip process we count the dislocations comprised in this thickness.

The commonly used stick-slip model, together with the elastodynamic equation, eliminates the self-stress fields from the objects on a fault plane. In our approach the self stresses in interaction with elastic stresses govern a slip evolution process.
On a fault $(x, t)$ the dynamic processes cause a rapid increase in the self-stress fields expressed by formation of dislocations and dislocation arrays (cracks - in the fracture phase). The total stresses can be split into elastic stress field and self-stress field, which for this case correspond to wave field and dislocation stress field: $S^{T}=S+S^{S}$. For dislocation density $\alpha$, dislocation velocity $V$ and also for the selfstresses, we may assume, according to condition (2.1), a rapid decrease away from the fault plane

$$
\begin{aligned}
& \alpha(x, y, t)=\exp \left[-a y^{4}\right] \vec{\alpha}(x, t) \\
& V(x, y, t)=\exp \left[-a y^{4}\right] \vec{V}(x, t) \\
& S^{s}(x, y, t)=\exp \left[-a y^{4}\right] \vec{S}(x, y)
\end{aligned}
$$

where $a$ is a constant.

The dislocation velocity can be also related to the local stress field (Mataga et al., 1987). Finally, for the near fault zone we come to the following equations for dislocation density (the $1 \mathrm{D}$ case $\{x, t\}$; Teisseyre and Yamashita, 1999; Teisseyre, 2001a):

$$
\frac{1}{c} \frac{\partial \alpha}{\partial t}=-\frac{\partial \alpha}{\partial x}+\Pi
$$

where $c$ is shear wave velocity.

The dynamic evolution is related to the dislocation fields and it depends mainly on the source/sink function $\Pi$. This function describes the nucleation of the new dislocations and the coalescence processes (mutual annihilation of dislocations of the opposite signs - the process equivalent to coalescence of two neighboring dislocated elements or formation of a crack). The source/sink function shall first of all describe the coalescence processes between the dislocation arrays of the opposite signs (the coalescence of the arrays well describes a nucleation process of a crack - material fracturing). We may relate this function to a stress 
surface curvature, or a gradient of dislocation density (inverse of a mean distance between the opposite dislocation groups). Attraction forces between the opposite dislocations can effectively produce a weakening effect, and in this sense the function $\Pi$ acts equivalently to the friction weakening laws.

For a review related to source/sink function $\Pi$, see the paper by Teisseyre and Nagahama (1998).

Here, we use the modified form of this function (Teisseyre, 2001b) in terms of dislocation density

$$
\Pi=\Pi^{0}\left[\alpha\left(\frac{\partial}{\partial x} \alpha\right)^{2}-\varepsilon \alpha^{2} \frac{\partial}{\partial x} \alpha\right] .
$$

The first term describes the formation process of new small elements of dislocations (stresses decrease as $1 / r^{2}$ ) and proportional to dislocation density $\alpha$ and to square of stress curvature, while the second term describes coalescence process of the opposite dislocation lines (stresses decrease as $1 / r$ ) and proportional to square of dislocation density $\alpha^{2}$ and to stress curvature; $\varepsilon$ is a constant.

\section{Numerical simulations of dislocation evolution}

Following the paper by Teisseyre (2001b) we briefly present the results of the numerical simulations of dislocation evolution, assuming that a micro-source is represented by a microdomain $\{x\},\{t\}$ of the dimensions $\left\{0,2 \pi \cdot 10^{-3}[\mathrm{~m}]\right\}$, $\left\{0,1.6 \cdot 10^{-5}[\mathrm{~s}]\right\}$, and the initial conditions chosen as follows:

$$
\begin{gathered}
\alpha(x, 0)=[0.05-0.2 \sin (x-\pi)] \cdot 10^{6}\left[\frac{1}{\mathrm{~m}}\right] \\
\alpha(0, t)=\alpha(2 \pi, 0) .
\end{gathered}
$$

According to eq. (2.5) and definition (2.6), we obtain the equation

$$
\begin{gathered}
\frac{\partial \alpha(x, t)}{\partial t}+ \\
+10^{-2}\left(0.41-0.1 \cdot 10^{-6}|\alpha(x, t)|\right) \frac{\partial|\alpha(x, t)|}{\partial x}+ \\
+1.5 \cdot 10^{-11}|\alpha(x, t)| \alpha(x, t)= \\
-4.63951 .5 \cdot 10^{-13} \alpha(x, t) \frac{\partial \alpha(x, t)}{\partial x} . \\
\cdot\left(|\alpha(x, t)|-10^{-3}\left|\frac{\partial \alpha(x, t) \mid}{\partial x}\right|\right) .
\end{gathered}
$$

The numerical simulations show some instabilities in time which might be identified with the microseismic events acting in a premonitory domain. The computed fields remain much smoother along the $x$-axis.

\section{Electric current and electromagnetic field at micro-source}

The electric polarization induced by dislocation processes depends on the square of dislocation density, because both the electric charge and the coalescence break distance between the dislocations are proportional to dislocation density $\alpha$. For polarization density and its derivatives we obtain

$$
p=\frac{1}{2} \kappa \alpha^{2}, \quad \dot{p}=\kappa \alpha \dot{\alpha}, \quad \ddot{p}=\kappa\left(\alpha \ddot{\alpha}+\dot{\alpha}^{2}\right)
$$

while, according to Ogawa et al. (1985), for electromagnetic exitations we have the following formulae expressing the near or electrostatic field, as well as the induction and radiation electric fields related to the dipoles activated in rocks

$$
\begin{gathered}
E^{\text {near }}=\frac{1}{4 \pi \varepsilon_{0}} \frac{p}{r^{3}}, \quad E^{\text {ind }}=\frac{1}{4 \pi \varepsilon_{0}} \frac{\dot{p}}{c_{0} r^{2}}, \\
E^{\mathrm{rad}}=\frac{1}{4 \pi \varepsilon_{0}} \frac{\ddot{p}}{c_{0}^{2} r}
\end{gathered}
$$


where $\varepsilon_{0}$ is the dielectric constant; the time derivatives are related to the source $\left\{x_{i}^{0}\right\}$ and related activity time $t^{0} ; c_{0}$ is light velocity.

Of course, we are aware of the fact that the different processes related to excitation of the electromagnetic field take place in a precursory time domain and during an earthquake event (Shevtsova, 1984; Varotsos and Alexopoulos, 1984, 1986; Gokhberg et al., 1985; Varotsos et al., 1992; Park, 1993; Teisseyre, 1992, 1995). For review on the dislocation processes and formation of electric charges, see Whitworth (1975).

Here, we try to include also the electromagnetic emission from the pre-seismic microsources and to explain this emission by dislocation dynamic processes.

\section{Numerical estimations for electric current and electromagnetic radiation}

We refer to estimations presented by Teisseyre (2001b). An order of magnitude of dislocation density at the time of micro-fracturing may be compared to density at the tip of dislocation array. For the dislocation density treated as a product of line density and the Burgers vector (the definition used in theoretical papers) and referred here to the band width $\Lambda$ and the $n$ dislocations with the Burgers vector $\lambda_{0}$ (lattice constant), densely packed in an element $d_{0}=m \lambda_{0}$, we obtain

$$
\begin{gathered}
\alpha=\frac{n \lambda_{0}}{d_{0} \Lambda}, \quad \alpha=\frac{n \lambda_{0}}{m \lambda_{0} \Lambda}<\frac{1}{\Lambda}, \\
\alpha^{\max } \approx 10^{6}\left[\mathrm{~m}^{-1}\right]
\end{gathered}
$$

while for the density of dislocation lines (the definition of line density used in papers on laboratory experiments, as a reference value we may cite its order for the elastic regime of a crystal body: $10^{11}\left[\mathrm{~m}^{-2}\right]$ )

$$
\bar{\alpha}=\alpha / \lambda_{0}
$$

$$
\begin{aligned}
& \bar{\alpha}=\frac{n}{m \lambda_{0} \Lambda} \ll \frac{1}{\lambda_{0} \Lambda} \\
& \bar{\alpha}^{\max }=10^{16}\left[\mathrm{~m}^{-2}\right] .
\end{aligned}
$$

The maximum of this density at local microfraturing $(n \rightarrow m)$ may theoretically approach $\bar{\alpha}^{\max }$ (corresponding to the line density at a crack tip).

The dislocation dynamics, as discussed in our model, can cause the appearance and activation of the electromagnetic dipoles in preseismic sources located in an earthquake preparation zone, but not exactly at the future main seismic spot. Based on numerical simulations for the evolution dislocation fields, we can present here the numerical simulation of the dipole intensities in the pre-seismic sources; the dipole source intensities as a function of time for the induction and radiation terms (4.1) are presented in figs. 1 and 2 in the following scales:

$$
\alpha\left(x_{i}, t\right) \dot{\alpha}\left(x_{i}, t\right) \text { in the scale } 10^{17}\left[\frac{1}{\mathrm{~m}^{2} \mathrm{~s}}\right]
$$

for $x_{i}=1,2,3,4 \cdot 10^{-3}[\mathrm{~m}]$ and $\{t\}$ in the scale $\left[10^{-5} \mathrm{~s}\right]$,

and

$\dot{\alpha}^{2}\left(x_{i}, t\right)+\alpha\left(x_{i}, t\right) \ddot{\alpha}\left(x_{i}, t\right)$ in the scale $10^{22}\left[\frac{1}{\mathrm{~m}^{2} \mathrm{~s}^{2}}\right]$

for $x_{i}=1,2,3,4 \cdot 10^{-3}[\mathrm{~m}]$ and $\{t\}$ in the scale $\left[10^{-5} \mathrm{~s}\right]$.

These electromagnetic phenomena evidently precede a seismic event; the sharp increases of the electric current source intensities both for the induction and radiation fields are observed.

For density electric current, or induction field in a microfracture source we obtain the estimate (Teisseyre, 2001b):

$$
j_{0}=\dot{p}=10^{-9} \alpha \dot{\alpha}\left[\mathrm{A} / \mathrm{m}^{2}\right]<10^{6}\left[\mathrm{~A} / \mathrm{m}^{2}\right]
$$

while for the source intensity of radiation field 


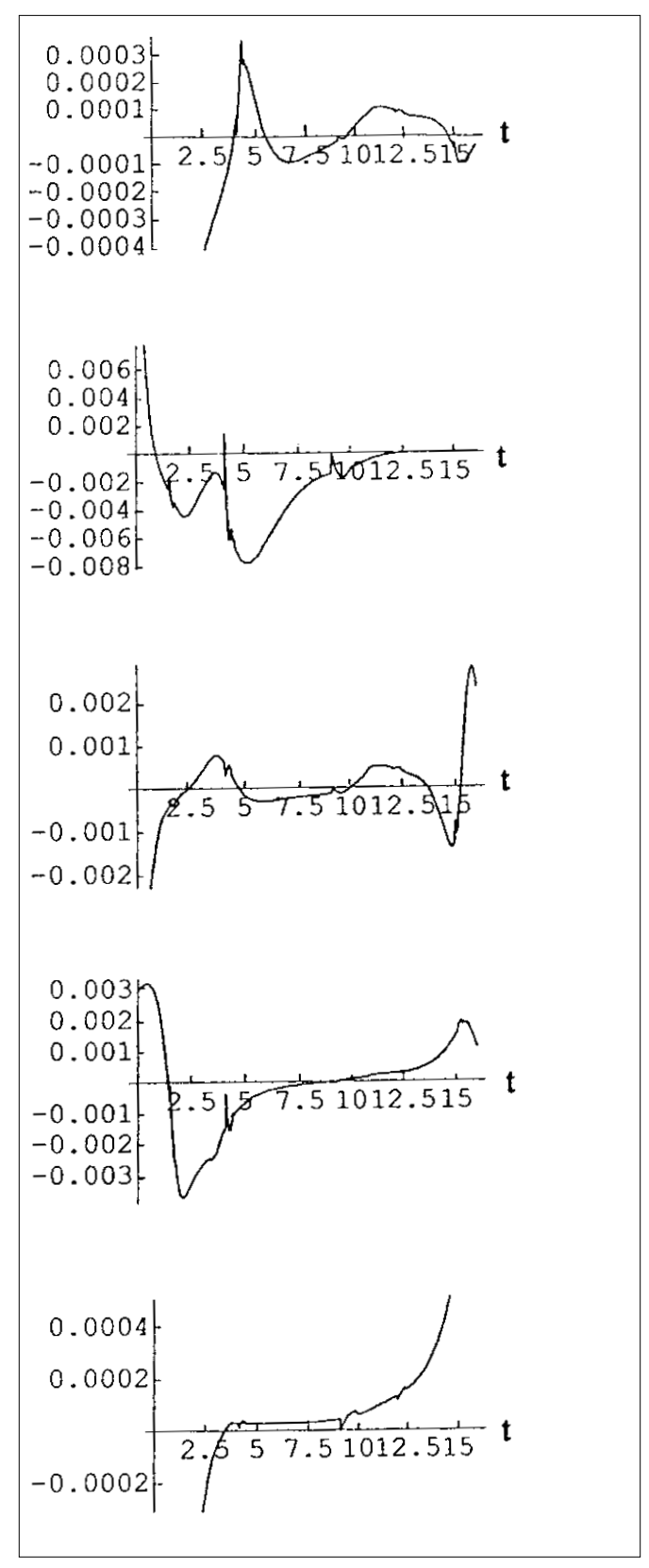

Fig. 1. Source intensities of electromagnetic induction field $\alpha(x, t) \dot{\alpha}(x, t)$ : the plots of $\alpha\left(x_{i}, t\right) \dot{\alpha}\left(x_{i}, t\right)$ in the scale $10^{17}\left[1 / \mathrm{m}^{2} \mathrm{~s}\right] ;$ for $x_{i}=1,2,3,4 \cdot 10^{-3}[\mathrm{~m}]$ and $\{t\}$ in the scale $\left[10^{-5} \mathrm{~s}\right]$.
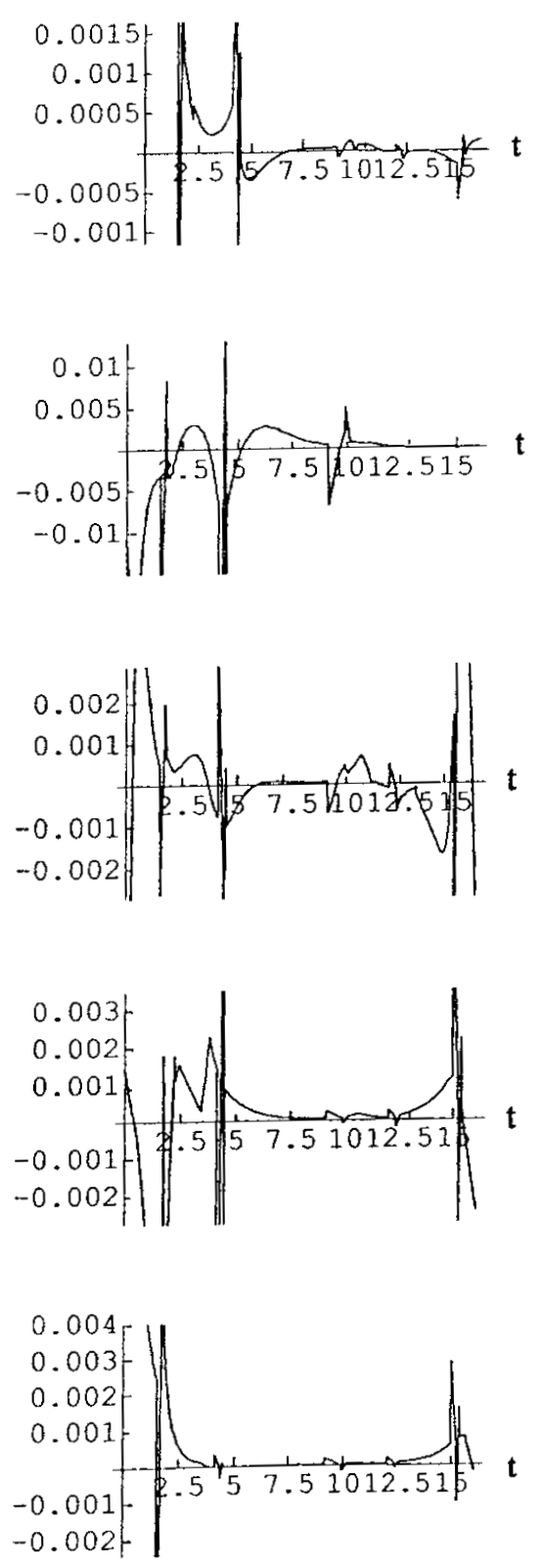

Fig. 2. Source intensities of electromagnetic radiation field $\dot{\alpha}^{2}(x, t)+\alpha(x, t) \ddot{\alpha}(x, t)$ : the plots of $\dot{\alpha}^{2}\left(x_{i}, t\right)+\alpha\left(x_{i}, t\right) \ddot{\alpha}\left(x_{i}, t\right)$ in the scale $10^{22}\left[1 / \mathrm{m}^{2} \mathrm{~s}^{2}\right]$; for $x_{i}=1,2,3,4 \cdot 10^{-3}[\mathrm{~m}]$ and $\{t\}$ in the scale $\left[10^{-5} \mathrm{~s}\right]$. 


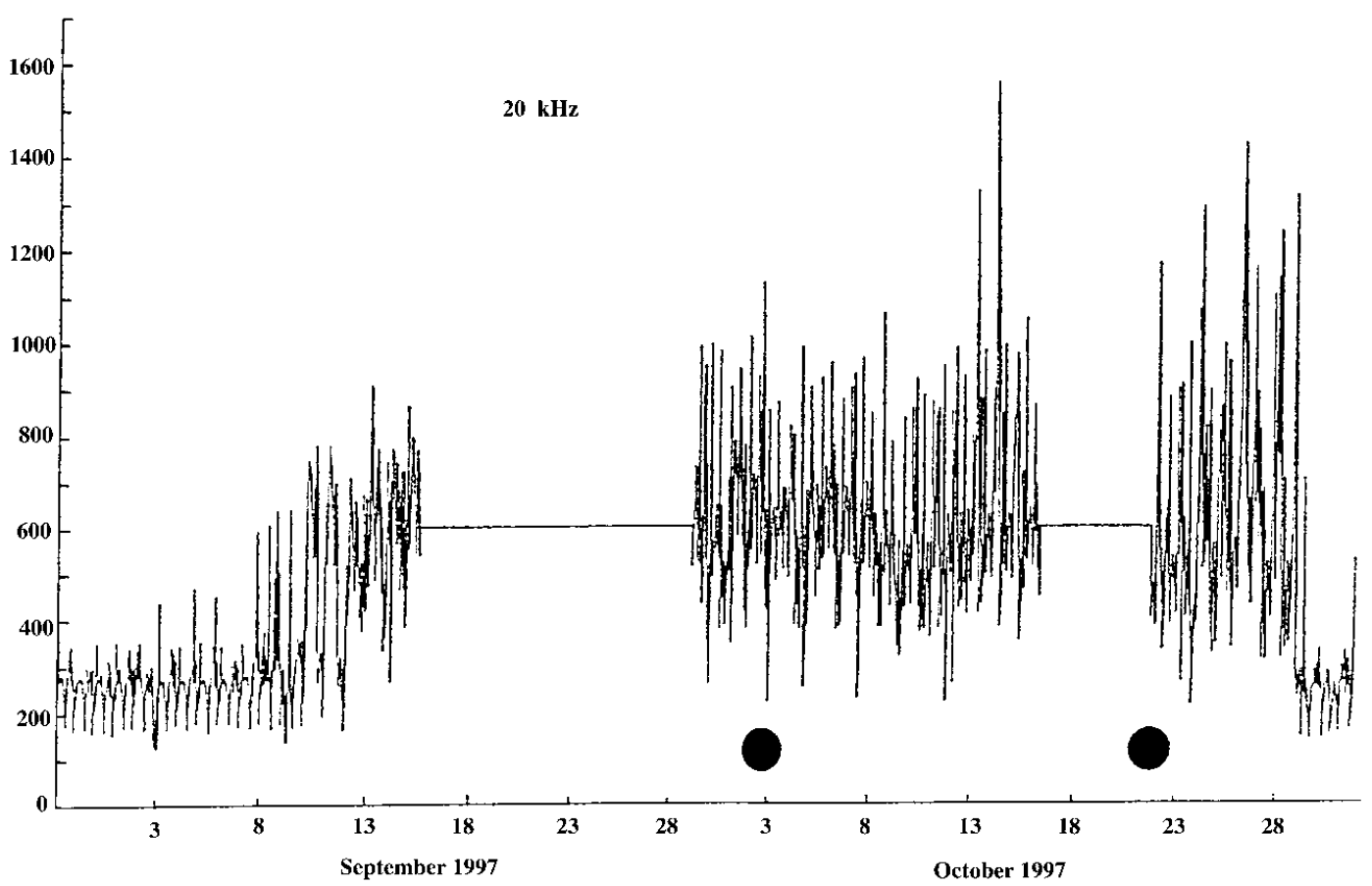

Fig. 3. VLF/recording $(20 \mathrm{kHz})$ of radio-noise at L'Aquila Observatory (after Meloni et al., 2001, and Ernst et al., 2001): September and October, 1997; two greater seismic events with $M \approx 5$ are marked by the black circles, straight intervals correspond to the gaps of recording.

we obtain

$\frac{\mathrm{d}}{\mathrm{d} t} j_{0}=\ddot{p}=10^{-9}\left(\dot{\alpha}^{2}+\alpha \ddot{\alpha}\right)\left[\mathrm{A} / \mathrm{m}^{2}\right]<10^{11}\left[\mathrm{~A} / \mathrm{sm}^{2}\right]$

Such intensity peaks may appear at the isolated small areas of the precursory microfracturings; activation of such local peaks of the $\alpha$ and $\dot{\alpha}$ fields causes the electromagnetic emission.

\section{Example of the observed electromagnetic excitations}

There exist numerous examples of radio-noise excitations before earthquake events; there, we present one example. At L'Aquila Geophysical Observatory (Central Apennines) a radio-noise recording system operating at frequencies of 10 , 20 and $40 \mathrm{kHz}$ has been installed (cooperation between the Institute of Geophysics, Polish Academy of Sciences, Warszawa, and the Istituto Nazionale di Geofisica e Vulcanologia, Roma).

Several seismic events with magnitudes over 4 and in some cases more than 5 were felt at the end of 1997 at distances of 60-80 km from the Observatory. In fig. 3 (Meloni et al., 2001; Ernst et al., 2001) we present the radio-noise recording (here for $20 \mathrm{kHz}$; similar records have been obtained for other frequencies) for the time when these seismic events occurred (marked on the plots). We shall note that for several years since the station was installed no such signals have appeared in the radio-noise observations; throughout this time, the seismicity was very low, with earthquakes reaching magnitudes of only $M \approx 3$. 


\section{REFERENCES}

ERnst, T., J. JANKOWSKi, R. TEISSEYRE, A. MELONI and P. PALANGIO (2001): Observations of electromagnetic radiation in the Central Apennines seismic region, Acta Geophys. Pol., 49, 43-53.

GOKHBERG, M.B., I.L. GUFELD, N.I. GERSHENZON and V.A. PILIPENKO (1985): Electromagnetic effects during rupture of the Earth's crust, Izv. Akad. Nauk SSSR, Fiz. Zemli, 1, $72-87$ (in Russian); Izv. Earth Phys., 21 (1), 52-63.

KosseckA, E. and R. DEWitT (1977): Disclination kinematic, Arch. Mech., 29, 633-651.

MataGA, P.A., L.B. FreUnd and J.W. HutChinson (1987): Crack tip plasticity in dynamic fracture, J. Phys. Chem. Solid, 48, 985-1005.

Meloni, A., D. Di Mauro, G. Mele, P. Palangio, T. ERnst, and R. TEISSEYRE (2001): Evolution of magnetotelluric, total magnetic field, and VLF field parameters in Central Italy: relations to local seismic activity, Ann. Geofis., 44 (2), 383-394.

OGAWA, T., K. OIKE and T.MiURA (1985): Electromagnetic radiations from rocks, J. Geophys. Res., 98 (D4), 6245-6249.

PARK, S.K., M.S. Johnston, T.R. MAdDEN, F.D. MoRGan and F.F. MORRISON (1993): Electromagnetic precursors to earthquakes in the ULF band; a review of observations and mechanisms, Rev. Geophys., 31 (2), 117-132.

ShEVTSOVA, I.N. (1984): Charging of dislocations during deformation of crystals with ionic-type bonds, Izv. Earth Phys., 20 (8), 643-648.

TEISSEYRE, R. (1992): Earthquake premonitory processes: Evolution of stresses and electric current generation, Terra Nova, 4, 509-513.

TEISSEYRE, R. (1995): Electric field generation in earthquake premonitory process, in Theory of Earthquake
Premonitory and Fracture Processes, edited by R. TEISSEYRE, 282-292, PWN, Warszawa.

TEISSEYRE, R. (1996): Motion and flow equation for stresses, Acta Geophys. Pol., 44, 19-29.

TEISSEYRE, R. (2001a): Evolution, propagation and diffusion of dislocation fields, in Earthquake Thermodynamics and Phase Transformations in the Earth's Interior, edited by R. TEISSEYRE and E. Majewski (Academic Press, San Diego, San Francisco, New York, Boston, London, Sydney, Tokyo), International Geophysical Series, vol. 76, 167-198.

TEISSEYRE, R. (2001b): Dislocation dynamics and related electromagnetic excitation, Acta Geophys. Pol., 49, 55-73.

TEISSEYRE, R. and H. NAGAHAMA (1998): Dislocation field evolution and dislocation source/sink function, Acta Geophys. Pol., 46, 13-33.

TEISSEYRE, R. and T. YAMASHITA (1999): Splitting stress motion equation into seismic wave and fault-related fields, Acta Geophys. Pol., 47, 135-147.

Varotsos, P. and A. Alexopoulos (1984): Physical properties of the variations of the electric field of the earth preceding earthquakes. II - Determination of epicenter and magnitude, Tectonophysics, 110, 99-125.

VARotsos, P. and A. Alexopoulos (1986): Thermodynamics of Point Defects and their Relation with Bulk Properties (North-Holland Publ. Comp., Amsterdam New York), pp. 474.

VARotsos, P., N.G. Bogris and A. KYRITSIS (1992): Comments on the depolarization currents stimulated by variations of temperature or pressure, J. Phys. Chem. Solids, 53 (8), 1007-1011.

WhitworTH, R.W. (1975): Charged dislocations in ionic crystals, Adv. Phys., 24 (2), 203-304. 